Archives

18-19 | 1997

Inventaire des archives de Robert Mandrou

\title{
Une expérience intellectuelle
}

\section{Arlette Farge}

\section{(2) OpenEdition}

Journals

\section{Édition électronique}

URL : http://journals.openedition.org/ccrh/2561

DOI : $10.4000 /$ ccrh.2561

ISSN : $1760-7906$

Éditeur

Centre de recherches historiques - EHESS

Édition imprimée

Date de publication : 10 octobre 1997

ISSN : 0990-9141

Référence électronique

Arlette Farge, "Une expérience intellectuelle », Les Cahiers du Centre de Recherches Historiques [En ligne], 18-19 | 1997, mis en ligne le 20 février 2009, consulté le 22 avril 2019. URL : http:// journals.openedition.org/ccrh/2561 ; DOI : 10.4000/ccrh.2561

Ce document a été généré automatiquement le 22 avril 2019

Article L.111-1 du Code de la propriété intellectuelle. 


\title{
Une expérience intellectuelle
}

\author{
Arlette Farge
}

1 Robert Mandrou, en séminaire, explorait, devant son public de vastes chantiers d'histoire qui jusqu'alors n'avaient jamais été exploités ni même répertoriés. Participer à son enseignement du samedi matin, rue de Varenne, demandait d'entrer de façon exigeante dans une démarche neuve, aux problématiques en train de s'élaborer. Le métier, c'est-àdire l'ensemble de ses questions, de ses procédures et surtout de ses enjeux, prenait corps de façon concrète alors même qu'il était constamment sous-tendu de théorisations et de grilles d'interprétation savantes. La démarche était dynamique, le rythme toujours; quant à la parole de Robert Mandrou, elle était aiguë et ironique, rigoureuse et patiente.

Plusieurs publics étaient présents: aux étudiants en thèse se mêlaient de jeunes chercheurs ainsi que des historiens ou historiennes déjà en poste. Les plus jeunes, les moins informés, recevaient là une formation qui respectait toutes les étapes du métier d'historien et englobait l'histoire des champs historiographiques. Dans la façon d'enseigner de Robert Mandrou se logeaient avec précision les raisons de ses questionnements, celles de ses conflits avec d'autres intellectuels, les méandres de ses recherches, l'analyse des documents, les moyens d'aller aux sources (notamment la découverte des archives judiciaires), les avancées de son travail. En filigrane se transmettaient les leçons apprises de ses maîtres et, par l'intermédiaire de ses choix personnels et éthiques qui furent aussi des refus, une leçon civique, éthique clairement affirmée.

3 La première heure était, la plupart du temps, consacrée à la critique d'un livre récent. En général, ce livre s'accordait aux thèmes discutés cette année-là en séminaire - Mandrou représentant à cette époque l'histoire des mentalités en train de se mettre en place, les ouvrages, les thèses ne manquaient pas d'être présents. Une vraie profusion intellectuelle semblait garnir cette première heure du séminaire; on pouvait avoir l'impression que l'époque était alors très féconde, généreuse, imaginative et libre aussi. Ce moment était pour le public le chemin de bien des découvertes; et Robert Mandrou les accompagnait d'un discours critique extrêmement fin, appuyé, pour certains acerbe, pour d'autres librement salutaire. Le savoir offert ici était ancré dans une vaste érudition, sans être présenté comme quelque chose de définitif ou de donné pour toujours. À travers les 
ouvrages présentés, Robert Mandrou disait quelque chose de ses choix, de ses convictions; il décortiquait avec aisance les résultats acquis pour mettre certains en doute, pour préparer de nouvelles pistes de recherche aux étudiants et aux chercheurs, pour tracer une voie personnelle dans l'ensemble des grands sujets dont il était l'initiateur (soulèvements populaires, médecine, sorcellerie, criminalité, etc.), pour rendre grâce aussi aux chemins déjà parcourus. Cette première heure de séminaire ressemblait en quelque sorte à un marathon : le professeur, en développant « la course » des auteurs qu'il présentait, offrait une autre course, d'autres chemins, d'autres questions à ceux qui l'entendaient. Un livre choisi et critiqué était une étape, un moyen d'aller plus loin, à moins - et cela arrivait - qu'il ne soit clairement critiqué et mis en pièces. Cette fois-là, toutes les raisons intellectuelles de la critique étaient mises au jour les unes après les autres : au public alors de réfléchir et de prendre ses voies personnelles. Les champs historiographiques de l'époque étaient si nouveaux qu'il semblait bien, à cette heure-là, qu'il n'y avait rien de fermé. Et le «critiquable » devenait le moyen d'aller ailleurs, un ailleurs dans lequel chacun se trouvait guidé, s'il le désirait.

Cette particularité de tirer parti de tout ouvrage, de tout chantier historique pour ouvrir des pistes se retrouvait ailleurs, dans les publications de Robert Mandrou et dans ses grandes synthèses. À la fin de ses ouvrages (Histoire de la civilisation française, 1958, rédigée avec Georges Duby ; Introduction à la France moderne, essai de psychologie historique, 1961, contemporaine de l'Histoire de la folie de Michel Foucault ; La France aux XVII et XVIII siècles ), un dernier chapitre énumérait ce qui restait ouvert, ce sur quoi il était urgent de travailler et pourquoi. On peut encore les relire : tout n'a pas été fait.

5 La deuxième heure du séminaire portait sur les travaux personnels de Robert Mandrou. Aidé de notes détaillées, de feuillets manuscrits rédigés d'une plume fine, il exposait ses résultats, développait sa démarche personnelle en expliquant vers où elle allait et aussi contre quoi et contre qui. Aucune grande querelle historique (Porchnev, Mounier; histoire quantitative, histoire dite qualitative...) n'était mise de côté : le travail de Robert Mandrou s'en nourrissait pour avancer, exposer ses méthodes, tenter des résultats, répondre aux critiques (culture populaire, culture savante; sorcellerie), encourager les chercheurs à ouvrir de nouveaux chantiers eux aussi. Écouter cet enseignement, c'était en quelque sorte être déplacé par rapport à l'ordre habituel du savoir historique, comprendre que le débat est une forme essentielle de la vie intellectuelle, n'être jamais en état de réception passive mais poussé par plusieurs types d'élan critique. Il y avait là un enseignement, c'est-à-dire un savoir, mais aussi les moyens de critiquer ce savoir, de s'approprier une intelligence prospective pour soi, et au-delà de l'enseignement donné se profilait une personnalité délibérément républicaine qui marquait son travail et ses exigences éthiques. De grandes figures traversaient son discours : Lucien Febvre, Philippe Ariès, Georges Duby ; de grandes amitiés l'entouraient tandis qu'il était à fois discret mais visiblement profondément affecté et transformé par le conflit qu'il jugeait intolérable avec la personne de Braudel. L'enseignement donné ne transmettait jamais la gravité de cette querelle, pour ne délivrer que le goût de l'analyse et de la prospective.

Les travaux portaient sur des thèmes amples qui ont fait date : sorcellerie, corps, histoire culturelle, Bibliothèque bleue, criminalité). Ces thèmes relevaient d'une vision du monde spécifique. Quoi qu'il en ait été des critiques, et bien qu'on ait été obligé de se séparer de certaines interprétations données à l'époque, il n'a pu échapper à personne que toutes avaient été choisies à l'intérieur de questionnements sur les déplacements, les ruptures, les sensibilités et les marges. Ce n'était pas vraiment le monde à l'envers, mais il s'agissait 
bien d'une focalisation spécifique sur des univers inconnus révélant des sociétés marginalisées, populaires, réprimées. Cela paraît à présent banal. C'était simplement l'aube d'enquêtes nouvelles où déjà se recherchaient, au-delà de l'étude des grands systèmes, les voix et les individus qui avaient été blessés par ces systèmes ou qui s'étaient révoltés. Une histoire de l'accident, de la rupture, des chemins interrompus s'amorçait en même temps que celle d'un « univers mental » aux imaginaires et aux croyances, utiles à décoder et à comprendre.

7 C'est dans ce cadre que la direction d'études menée par Robert Mandrou prenait sens et se rattachait aux exigences de son séminaire. L'attention aux sources sur lesquelles travaillaient les chercheurs prenait une place prééminente : qu'il s'agisse de l'étude des textes imprimés dits non savants, ou de la grande investigation décidée à travers les archives judiciaires (en même temps que l'équipe de Pierre Chaunu à Caen), le soin accordé à la lecture particulière de « récits d'histoire » singuliers était particulièrement important. Lire des archives avec Robert Mandrou, c'était apprendre à construire immédiatement des grilles de lecture et à approcher qualitativement et scientifiquement un monde peu connu des historiens. Rusé, Robert Mandrou ne guidait guère vers les tentations trop évidentes de sensibilités exacerbées ou de lectures trop faciles d'une réalité, qui en fait échappait toujours, puisqu'elle n'était pas - tant s'en faut - déployée dans l'archive. Réservé, pudique, Robert Mandrou organisait son séminaire et ses directions de travaux dans un espace fort, d'éthique, de réflexion et d'esprit critique. De cette trame solide s'échappaient humour, fidélité, et de piquants traits d'ironie. 